\title{
AVANT-PROPOS
}

\section{Recherche halieutique et gestion des pêches ${ }^{\star}$}

\author{
Serge M. Garcia ${ }^{a}$ \\ Division des Ressources Halieutiques, Département des Pêches de la FAO Viale delle Terme di Caracalla, 00100 Rome, Italie
}

Depuis 50 ans, le problème délicat posé par la relation entre la science et la gestion des ressources naturelles a été très souvent abordé et je dois donc traiter d'un thème fondamental sur lequel il est difficile d'être original. L'un des travaux français les plus récents et intéressants sur ce sujet a été publie $^{1}$ par Joseph Catanzano et Hélène Rey en 1997. Centrée sur l'halieutique (comprise comme incluant toutes les disciplines nécessaires à une gestion moderne de la pêche), cette présentation décrit cependant des problèmes et des évolutions communes à toutes les branches de la science dédiée à la gestion rationnelle des ressources naturelles renouvelables ainsi qu'à l'analyse et la gestion des risques de société.

Après avoir passé en revue quelques-unes des principales questions relatives au processus impliquant l'halieutique dans les mécanismes de décision, nous mentionnerons brièvement les nouveaux concepts de gestion auxquels l'halieutique doit faire face et terminerons par quelques considérations générales sur l'évolution de la recherche.

\section{Quelques réflexions générales}

Examinons brièvement quelques-unes des questions fondamentales qui se posent actuellement.

\subsection{La contribution de la science au développement durable}

Le Chapitre 35 de la Conférence des Nations Unies pour l'Environnement et le Développement (CNUED) indique ce que devrait être la contribution de la science au développement durable. La liste des questions à traiter est longue : analyse des perspectives à long terme, intégration des échelles

* Article présenté au 6 ${ }^{\mathrm{e}}$ Forum Halieumétrique, 24-26 juin 2003, Montpellier, Faculté des Sciences Economiques.

a Auteur correspondant : serge.garcia@fao.org

${ }^{1}$ La recherche halieutique entre science et action : réflexions sur fond de crise. J. Catanzano et H. Rey, 1997, Nature Sciences et Sociétés vol. 5, n 2, 19-30. de temps et d'espace, utilisation des savoirs non conventionnels, incertitude, risque et précaution, évaluation et conservation de la capacité biologique, santé des écosystèmes, dégradation de l'environnement, changement du climat, gestion des usages multiples, déficit de communication, science et décision, interdisciplinarité, participation, coordination, partenariats, modèles de consommation, incitations, comportements, audit et contrôle, éveil des consciences, transparence, responsabilité, développement des capacités, systèmes d'information et échange de données, banques de savoir, infrastructures de recherche et conditions de vie des chercheurs.

Il est clair que la société attend beaucoup de la science pour mieux comprendre les ressources, améliorer leur conservation, optimiser leur utilisation, transformer les institutions, etc.

\subsection{Glissement de la demande sociale}

Le rôle spécifique de l'halieutique dans la gouvernance des pêches est enchâssé dans le Nouveau Droit de la Mer de 1982 qui dispose que les décisions doivent prendre en compte les «meilleures informations scientifiques disponibles ».

$\mathrm{Au}$ cours des 50 dernières années, l'halieutique s'est adaptée à l'évolution du secteur et du paradigme de gestion, réduisant progressivement sa contribution à la découverte des ressources nouvelles et à l'expansion du secteur pour se concentrer sur sa gestion et celle des usages multiples ainsi que sur l'analyse des performances, des interactions, des impacts sur l'environnement, de l'incertitude et des risques qui en découlent et, dans une moindre mesure, des stratégies de transition.

Ce glissement s'accélère et il est accompagné de tensions et de frictions dues à la rigidité des attitudes des acteurs et des institutions.

\subsection{Science et décision}

L'intervention de l'halieutique dans les processus de décision est inscrite dans les textes. A ce titre, l'halieutique relève en partie de l'expertise et requiert, entre autres : (i) une plus 
grande sensibilité aux objectifs sociaux et politiques, (ii) des réponses obligatoires et quasi immédiates, quel que soit la question et le niveau de compréhension, (iii) l'intégration des disciplines dans une analyse systémique, (iv) l'analyse de l'incertitude et de ses implications en termes de risques pour la ressource et le secteur, (v) une analyse des mécanismes et des implications des schémas d'allocation des ressources.

La responsabilité est lourde. Elle a favorisé l'acquisition d'un certain pouvoir (en partie fictif) et une mobilisation de moyens de recherche importants (encore souvent insuffisants). Elle a créé également des obligations de service qui ont souvent entravé son évolution et parfois entaché sa réputation.

L'interaction entre halieutique et décision améliore la crédibilité des décideurs, tout en réduisant leur espace de liberté. Bien que dans un nombre croissant de pays, ces derniers demandent à connaître l'ensemble des options disponibles et les incertitudes qui les entachent; les décideurs sont en général, mal à l'aise devant l'éventail des possibilités.

L'incapacité reconnue de la gestion à conserver les ressources a conduit à une remise en question du rôle de la science et de sa propre performance, créant une tension forte entre halieutes et décideurs, mettant en évidence ce que Catanzano et Rey ont appelé une «crise de proximité », caractérisée par :

- une érosion pernicieuse de l'objectivité sous la pression des employeurs;

- une recherche accrue de transparence comme une des garanties de l'indépendance scientifique;

- une dégradation de la qualité car les questions posées s'accumulent plus vite que les ressources et les données nécessaires pour y répondre correctement;

- une rigidité du paradigme opérationnel en décalage croissant avec les connaissances et le cadre conceptuel;

- un retard traditionnel de la recherche sur le développement technologique aggravé par un retard sur le développement institutionnel (par exemple en relation avec la précaution et l'approche écosystémique);

- une hypersensibilisation du système aux conséquences de l'incertitude scientifique sur les décisions qui en découlent ;

- des propositions pour une professionnalisation accrue du métier d'halieute;

- un accroissement des tensions entre les halieutes et les autres parties prenantes.

\subsection{Science et plaidoyer}

La science se veut neutre et vise à cerner la vérité, dans toute sa complexité, y compris les interprétations divergentes compatibles avec les données disponibles quand le consensus est impossible (situations d'indétermination scientifique). Elle est à la base de l'action gouvernementale dans les agences de gestion et suppose souvent un devoir de réserve.

Le plaidoyer (en anglais : advocacy) vise à convaincre le public et les hommes politiques qu'une des interprétations disponibles (celles qui conviennent le mieux aux intérêts particulier du plaideur) est la meilleure. Il est à la base de l'activisme de nombreuses Organisations non gouvernementales, ONG environnementales ou professionnelles.
La frontière entre ces deux activités sociales, parfaitement justifiables mais diamétralement opposées sur le plan de l'éthique scientifique, peut être facilement mais inopportunément franchie par les scientifiques, particulièrement quand les objectifs affichés par l'Etat manquent de clarté.

De nos jours, de nombreux scientifiques sont employés par des ONG pour défendre des positions spécifiques. L'implication croissante des scientifiques dans le plaidoyer peut conduire à une distribution plus équitable des connaissances scientifiques que par le passé. Cependant, l'effacement progressif des frontières entre science, plaidoyer et journalisme à sensation conduit à une confusion des genres et à un affaiblissement potentiel du crédit accordé par la société à la recherche.

Il est possible pour un scientifique de jouer les deux rôles à deux moments différents de sa carrière. Il est dangereux de les jouer simultanément.

\subsection{Du consensus scientifique à la polarisation partisane}

Il s'agit là d'une question symétrique de la précédente. La période de recherche active du consensus scientifique comme base de décision, qui a caractérisé le travail des commissions régionales des pêches depuis un demi-siècle touche, peut-être à sa fin, alors que se développe une tendance à la polarisation scientifique autour d'interprétations divergentes et conflictuelles des données disponibles.

La Commission Baleinière Internationale a été un précurseur en la matière, probablement à cause de l'intrusion dans le système de considérations éthiques fortement divergente sur la nature, le rôle, et l'utilisation des baleines. Le phénomène s'étend progressivement a d'autres instances lorsque des arguments non biologiques interfèrent (de manière souvent non transparente) avec le processus de décision.

Dans ce processus, l'incertitude est systématiquement recherchée, maintenue et même accrue lorsque cela convient pour jeter le doute sur des décisions considérées comme non favorables. Le problème est amplifié par les media.

D'un côté, on peut craindre que l'objectivité soit en cause et regretter le bon vieux temps du consensus. Mais de l'autre, il faut reconnaître que durant cette époque la science « consensuelle », essentiellement biologique, n'a eu que peu d'impact stratégique sur la conduite des pêches. La polarisation est peut-être l'indication que, le cadre de son action ayant évolué et l'incertitude étant formellement reconnue, l'avis scientifique aura des conséquences différentes pour les divers inserts concernés, rendant nécessaire une négociation. La science n'est plus le détenteur de la vérité mais un des acteurs privilégiés d'un processus social complexe et seulement partiellement rationnel.

\subsection{Science et communication}

Le triangle conventionnel (science - gestion - pêcheurs) devient de plus en plus complexe et de nouveaux flux d'information se sont crées :

- entre scientifiques et professionnels, développant un dialogue plus intense (au sein de commissions mixtes, par 
exemple) permettant de comparer perceptions et théories et de partager l'information. La collecte et l'utilisation explicite du savoir informel reste cependant limitée ;

- entre les scientifiques, l'administration et le public (y compris à travers les media) permettant d'instaurer une plus grande transparence, d'effectuer une meilleure évaluation des performances, et de faciliter l'expression des réactions du public et des consommateurs;

- entre scientifiques et gestionnaires, pour clarifier les objectifs, attentes et contraintes ; mieux s'informer mutuellement sur les options disponibles, les incertitudes qui les entachent et les risques qui en découlent.

- entre les scientifiques eux-mêmes, à travers les disciplines, pour mieux circonscrire objectivement l'incertitude, développer le consensus et réduire la polarisation.

Deux nouveaux flux prennent de l'importance : vers les media (à cause de la préoccupation publique croissante vis-àvis des pêches et de leur impact) ainsi que vers les tribunaux (à cause de la tendance au litige).

La conséquence directe de la polarisation scientifique (et politique), quand les mécanismes prévus pour la résolution des conflits sont inopérants, est l'accroissement rapide des recours aux tribunaux. Au niveau national, les tribunaux semblent être devenus, dans certains pays, le moyen le plus fréquent de «communication» et de «dialogue», ou la phase ultime de toute négociation quand on n'a pas obtenu gain de cause. Au niveau régional, le recours aux tribunaux par les commissions des pêches reste encore rare mais la soumission du cas du thon rouge du Sud Pacifique au tribunal du Droit de la Mer est un précurseur.

Si la tendance se confirme et se généralise, les futurs halieutes doivent se préparer à défendre leurs recommandations devant les tribunaux, dilapidant pour ce faire les ressources que la recherche devrait destiner à l'amélioration des connaissances et à la réduction de l'incertitude, source des conflits).

\subsection{Le coût de la recherche}

A priori, la relation entre les budgets de recherche et la qualité de l'aménagement ne parait pas très bonne. Des systèmes scientifiques sophistiqués sont impliqués dans des effondrements dramatiques alors que de bons résultats ont été obtenus avec une recherche minimale. Il a été noté également que pendant des siècles, les communautés traditionnelles ont été capables d'exploiter leurs ressources de manière durable, sans l'aide d'une science formelle.

Il existe des alternatives évidentes entre l'approche monospécifique peu coûteuse mais limitée, l'approche multispécifique plus complète mais plus gourmande en ressources, l'approche écosystémique, idéale mais plus encore complexe et plus coûteuse. Les approches les plus complètes sont aussi celles impliquant le plus haut niveau d'incertitude. Toutes ces approches peuvent être mises en œuvre de manière rigide ou adaptative, de manière simple ou compliquée et avec des résultats plus ou moins précis et/ou fiables.

Le problème est que l'on tend à demander à la recherche de faire mieux et en plus sans ressources supplémentaires. Dans de nombreux pays en développement, la situation est littéralement dramatique. Certains travaux récents soutiennent par exemple que l'approche écosystémique n'est tout simplement pas applicable dans les pays en développement, faute de moyens.

Un effort substantiel est nécessaire pour développer des approches de la gestion moins gourmandes en recherche formelle, peut-être à travers une recherche participative et une meilleure utilisation des savoirs informels.

\subsection{Objectifs de gestion}

Les scientifiques se plaignent chroniquement du fait que les gestionnaires acceptent rarement d'exposer explicitement les objectifs vers lesquels ils tendent ou se contentent de présenter une liste d'objectifs plus ou moins standard et contradictoires. Dans ce domaine, un peu de pragmatisme est nécessaire. Il a déjà été noté par le passé que les objectifs des politiques, gestionnaires, chercheurs, pêcheurs, environnementalistes, sont le plus souvent en conflit. Ils sont donc plus ou moins partiellement dissimulés par nécessité dans une interaction largement basée sur la négociation.

Il me paraît donc clair que, malgré la quête incessante des objectifs, les scientifiques devront encore pour quelque temps opérer dans un cadre de décision partiellement opaque. Dans ce contexte, l'adoption de systèmes d'indicateurs de durabilité avec leurs valeurs de référence devrait promouvoir l'expression plus claire des objectifs.

\subsection{Savoirs informels}

L'intégration des savoirs informels est nécessaire sur les plans idéologique et pratique. Sur le plan idéologique, l'appropriation des décisions par les parties prenantes à travers des processus de participation, délégation et décentralisation, implique que leur compréhension des choses soit prise en compte et harmonisée avec la compréhension scientifique. Sur le plan pratique, la demande d'élargissement du domaine de recherche halieutique, à budgets constants ou décroissants, implique l'utilisation de sources d'informations moins coûteuses.

Les approches post-modernes de la gestion restant fondées sur la science, le scientifique devra identifier les savoirs pertinents, pour les transformer en évidences scientifiques, essayant de réconcilier activement les discordances éventuelles.

\section{Les nouveaux concepts de gestion}

Le cadre conceptuel de la gestion des pêches est en évolution rapide avec, entre autres, l'intégration de l'approche de précaution, des indicateurs de durabilité et de l'approche écosystémique. Cette évolution pose à l'halieutique de nouveaux défis.

\subsection{L'approche de précaution}

L'approche de précaution est mentionnée dans l'Article 15 de la Déclaration de Rio. Elle vise à réduire les risques en encourageant l'analyse de l'incertitude et de ses implications, par 
exemple en termes de coûts potentiels des erreurs de gestion. En fonction du niveau de risque, on utilise des instruments préventifs ou correctifs, une gestion explicite du risque et, au-delà, des processus de négociation.

À ces approches correspondent des formes différentes de la recherche : d'abord conventionnelle et proche des sciences de l'ingénieur, puis analyse du risque. Au-delà, les processus sociaux prennent le pas sur la recherche. Cette zone à haut risque est considérée comme le domaine d'intervention d'une science dite « post-normale » où (je cite) la dictature des «faits tangibles » (informations dures, quantitatives) est progressivement supplantée par les systèmes de "valeurs 》 (informations molles, qualitatives), impliquant dans le processus de décision et dans l'analyse des performances un nombre toujours croissant de partie prenantes.

L'application actuelle de l'approche est encore balbutiante et limitée à la biologie. Beaucoup reste à faire pour introduire la précaution dans toutes les disciplines de l'halieutique et toutes les étapes du processus de décision.

\subsection{Indicateurs de durabilité}

Les systèmes de référence pour le développement durable (SRDD) sont généralement construits autour d'indicateurs, d'objectifs et de contraintes avec des valeurs cible, limite et seuil, des échelles et des jugements de valeur. Leur usage est recommandé dans l'Agenda 21, Chapitre 40. Les spécificités de leur application aux pêches ont été étudiées des points de vue scientifique et opérationnel mais l'expérience pratique est encore limitée.

Ces systèmes représentent un appui potentiel précieux pour : (1) le suivi des progrès réalisés en matière de développement durable, (2) la clarification des objectifs de gestion, (3) l'analyse comparative des performances, (4) améliorer transparence et communication. Ils représentent un mode efficace d'organisation et de diffusion de l'information scientifique dans son cadre décisionnel et permettent d'intégrer les pêches dans des cadres de développement plus larges.

Elaborer et tester ces systèmes représente un vrai défi pour la recherche.

\subsection{L'approche écosystémique}

Une exigence forte se développe demandant l'intégration de la gestion des pêches dans celle de l'écosystème qu'elle exploite, prenant en compte les usages multiples de cet écosystème, les modifications des flux trophiques, les destructions des habitats, les dérives génétiques, les pollutions d'origine continentales, l'altération de la capacité biologique qui en découle, la variabilité naturelle, le changement de climat.

Reposant sur une philosophie des relations entre l'Homme et la Nature, l'approche écosystémique est un concept antique, négligé pendant des décennies, puis ramené au premier plan par le spectacle de la dégradation ambiante. La forme moderne du concept est en cours de développement et requiert, selon la position où l'on se place, une évolution progressive ou une révolution brutale de la gestion conventionnelle. Il est difficile aujourd'hui de prédire si l'évolution qui est en cours sera assez rapide et efficace pour éviter les frais d'une révolution sans nul doute difficile et coûteuse.

L'approche écosystémique des pêches (plus que la gestion conventionnelle) a besoin de l'halieutique mais, par sa complexité, elle implique un processus de décision essentiellement public impliquant des choix d'ordre privé.

\section{Conclusion : crises de fonctionnement et évolution des institutions}

Les spécialistes de la recherche institutionnelle ont décrit l'évolution cyclique des institutions à travers les phases suivantes : (i) développement des politiques face à la problématique du moment, (ii) mise en œuvre de ces politiques et développement des institutions, (iii) reconnaissance progressive de leurs défauts à travers des échecs répétés, (iv) crise institutionnelle après reconnaissance formelle des échecs, (v) recherche de nouvelles voies et solutions, etc.

Examinant l'histoire de la pêche et de sa gestion, nous semblons être a la fin d'un cycle commencé en 1946 avec la conférence internationale sur la surexploitation, dominé par la technologie et le profit. Nous sommes au début d'un nouveau cycle mieux équilibré, plus concerné par l'écosystème et l'intérêt public. Dans cette nouvelle boucle, la recherche a obtenu de jouer un rôle fondamental, en dépit de ses faibles performances dans le cycle précèdent.

Saura-t-elle surmonter les causes de son premier échec?

\subsection{De la recherche alibi à la recherche action}

Dans la première partie de cette présentation, nous avons passé en revue quelques-unes des grandes questions et indiqué quelques éléments de réponse. Certaines des solutions ne relèvent que de la recherche elle-même. Les plus stratégiques, cependant, impliquent des changements importants au niveau des autorités de tutelle, des processus de décision, de l'implication des sociétés civiles sans lesquels l'halieutique ne pourra pas évoluer.

Le processus de Recherche et Décision doit devenir de plus en plus transparent, adaptatif, multidisciplinaire et participatif. L'halieute doit se préparer, entres autres, à un rôle croissant des parties prenantes dans le financement, la programmation et l'évaluation de la recherche ainsi que dans la prise de décision, à travers des processus fortement médiatisés et, malheureusement, un recours croissant aux tribunaux.

L'éthique ainsi que les besoins de qualité, transparence et responsabilité impliquent que les fonctions de conseil, d'audit et de plaidoyer soient entreprises par des institutions différentes, sans confusion des genres.

Le poids relatif de la science dans la décision finale devrait diminuer au fur et à mesure que s'accrô̂ssent l'incertitude, la participation et la négociation. Paradoxalement, cependant, si l'on passe enfin de la recherche-alibi à une véritable rechercheaction, l'impact réel de l'halieutique sur la pêche devrait s'accroître de manière significative. 